\title{
Characterization of HAF-4- and HAF-9- localizing organelles as distinct organelles in Caenorhabditis elegans intestinal cells
}

Takahiro Tanji', Kenji Nishikori', Syoko Haga', Yuki Kanno', Yusuke Kobayashi', Mai Takaya', Keiko Gengyo-Ando 2,3, Shohei Mitani ${ }^{2}$, Hirohisa Shiraishi ${ }^{1}$ and Ayako Ohashi-Kobayashi ${ }^{1^{*}}$

\begin{abstract}
Background: The intestinal cells of Caenorhabditis elegans are filled with heterogeneous granular organelles that are associated with specific organ functions. The best studied of these organelles are lipid droplets and acidified gut granules associated with GLO-1, a homolog of the small GTPase Rab38. In this study, we characterized a subset of the intestinal granules in which HAF-4 and HAF-9 localize on the membrane. HAF-4 and HAF-9 are ATP-binding cassette $(\mathrm{ABC})$ transporter proteins that are homologous to the mammalian lysosomal peptide transporter TAPL (transporter associated with antigen processing-like, $A B C B 9$ ).
\end{abstract}

Results: Using transgenic worms expressing fluorescent protein-tagged marker proteins, we demonstrated that the HAF-4- and HAF-9-localizing organelles are not lipid droplets and do not participate in yolk protein transport. They were also ruled out as GLO-1-positive acidified gut granules. Furthermore, we clarified that the late endosomal protein RAB-7 localizes to the HAF-4- and HAF-9-localizing organelles and is required for their biogenesis.

Conclusions: Our results indicate that the HAF-4- and HAF-9-localizing organelles are distinct intestinal organelles associated with the endocytic pathway.

Keywords: ABC transporter, Caenorhabditis elegans, Intestine, Lipid droplet, Lysosome, Yolk protein, Endosome, Rab7

\section{Background}

The intestine of Caenorhabditis elegans is a multifunctional organ involved in the uptake, metabolism, storage, and distribution of nutrients, as well as in detoxification and immune defense [1]. It consists of a tube comprising only 20 epithelial cells that are not replaced after embryonic development; thus, each intestinal cell is considered to have multiple functions throughout the life of the animal. The intestinal cells are filled with heterogeneous granular organelles that are associated with specific organ functions [2]. Therefore, to understand how these individual cells achieve multiple intestinal functions, these granular organelles should be classified and their physiological roles determined. However, the relationships between different types

\footnotetext{
* Correspondence: aohashi@iwate-med.ac.jp

${ }^{1}$ Department of Immunobiology, School of Pharmacy, Iwate Medical University, 2-1-1 Nishi-tokuta, Yahaba, Shiwa-gun, Iwate 028-3694, Japan
} Full list of author information is available at the end of the article of granules, their physiological roles, and the regulation of their biogenesis are largely unknown.

The best studied of the intestinal granular organelles are the acidified organelles known as "gut granules", which are easily recognized by the presence of autofluorescent materials $[3,4]$. Gut granules are regarded as lysosome-related organelles, because GLO-1 localizes to embryonic gut granules, and is required for the biogenesis of the organelles. GLO- 1 is a member of the RAB family of small $G$ proteins and a homolog of mammalian Rab38; it is required for the biogenesis of melanosomes (lysosome-related organelles in melanocytes). Several other genetic factors are required for gut granule biogenesis [5-9]. Although some of the physiological roles of gut granules have been identified, such as the storage of zinc [10] and cholesterol [11], and signaling for aging [12], their overall functions remain poorly understood. 
Other well-studied intestinal granules are the fat storage organelles known as lipid droplets [13]. The intestine is the major organ responsible for fat storage. Similar to mammalian lipid droplets, those of C. elegans are characterized by a phospholipid monolayer membrane [14]. The short-chain dehydrogenase DHS-3 and the triglyceride lipase ATGL-1 were recently identified as marker proteins that localize on the membrane of lipid droplets $[15,16]$.

Previously, we reported that HAF-4 and HAF-9, ATPbinding cassette $(\mathrm{ABC})$ transporter proteins that are homologous to the mammalian lysosomal peptide transporter TAPL (transporter associated with antigen processing like, ABCB9), localize to the membrane of a subset of intestinal granular organelles approximately $2 \mu \mathrm{m}$ in diameter from the late larval to adult stages [17]. HAF-4 and HAF-9 are involved in maintaining the normal formation of their localizing organelles, probably by forming a heterodimer as a functional unit of a transporter $[17,18]$. The colocalization of HAF-4, HAF-9, and LMP-1, a homolog of mammalian lysosome-associated membrane proteins (LAMPs), suggests their relevance to lysosomes. This interpretation is further supported by the observation that HAF-4 and HAF-9 localize to the enlarged vacuole formed in the mutant of $p p k-3$, a gene regulating the terminal maturation of lysosomes. Furthermore, HAF-4- and HAF-9-localizing organelles are neither acidified nor autofluorescent granules, and the mutants of haf-4 and haf-9 show the same or a greater number of acidified and autofluorescent granules as the wild-type, suggesting that the HAF-4- and HAF-9-localizing organelles are distinct from gut granules. We also reported that the HAF-4- and HAF-9localizing organelles did not show positive vital staining for the lysochrome dye Nile Red; however, a subsequent study demonstrated that fixed Nile Red staining, but not vital staining, showed specificity to the lipid droplets in the cells $[13,14]$. Therefore, the possibility that HAF-4and HAF-9-localizing organelles are lipid droplets remained an open question.

Here, we report the classification of the HAF-4- and HAF-9-localizing organelles among intestinal granules using transgenic worms expressing fluorescent proteintagged marker proteins. We confirmed that these organelles are not lipid droplets and do not participate in yolk protein transport. They are also not GLO-1-positive gut granules. Further investigation using the late endosomal protein RAB-7 revealed that the HAF-4- and HAF-9localizing organelles are distinct intestinal organelles associated with the endocytic pathway.

\section{Results}

HAF-4- and HAF-9-localizing organelles are not lipid droplets

We determined whether the HAF-4- or HAF-9localizing organelles are lipid droplets by Nile Red staining of HAF-4::green fluorescent protein (GFP)and HAF-9::GFP-expressing transgenic worms following fixation of the worms in paraformaldehyde. Neither HAF-4-positive nor HAF-9-positive intestinal granules were stained with Nile Red (Additional file 1: Figure S1). We also compared the localization of HAF-9::mCherry with that of the lipid droplet markers DHS-3::GFP [15] and ATGL-1::GFP [16]. DHS-3::GFP did not localize to the surface of either the HAF-9::mCherry-positive granules (Fig. 1a) or the autofluorescent granules, which are regarded as acidified gut granules (Additional file 1: Figure S2). Furthermore, HAF-9::mCherry also did not localize to the surface of the ATGL-1::GFP-positive granules (Fig. 1b). These results indicate that the HAF-4- and HAF-9localizing organelles are not lipid droplets.

We next determined whether HAF-4 and HAF-9 are involved in the biogenesis of lipid droplets using deletion mutants of haf-4 and haf-9, which exhibit defects in the biogenesis of the HAF-4- and HAF-9localizing organelles [17]. These mutants did not show a decrease in the number of lipid droplets, as shown by Nile Red staining (Fig. 1c) and DHS-3::GFP localization (Fig. 1d), indicating that the organelles are not required for the biogenesis of lipid droplets. This conclusion was also supported by the normal formation of DHS-3::GFP-positive lipid droplets in the lmp-1 mutant, in which HAF-4- and HAF-9localizing organelles show morphological aberrations [17] (Additional file 1: Figure S3).

\section{HAF-4- and HAF-9-localizing organelles are irrelevant to yolk protein transport}

A previous report indicated that deletion mutants of haf-4 and haf-9 show a reduced brood size [17], which suggests the involvement of the HAF-4- and HAF-9-localizing organelles in the nutrition supply to oocytes. However, these organelles are found in both hermaphrodites and males (Fig. 2a and Additional file 1: Figure S13a). In C. elegans, VIT-2 (a yp170B homo$\log$ ) is secreted from the intestinal cells into the pseudocoelomic space, and is taken up by developing oocytes through gonadal sheath cells as a nutritional source [19]. VIT-2 is expressed only in the intestinal cells of the adult hermaphrodite. Although the mechanism by which the oocytes uptake yolk protein via receptor-mediated endocytosis is well characterized [20], precisely how the yolk protein is pooled and trafficked in the intestinal cells has not been elucidated in detail. The yolk protein in the intestinal cells was visualized by VIT-2::GFP, which was distinguishable from the autofluorescent granules and lipid droplets (Additional file 1: Figure S4). However, VIT2::GFP did not localize to either the HAF-9::mCherrypositive (Fig. 2b and Additional file 1: Figure S13b) or 
A

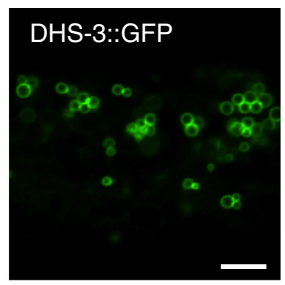

B

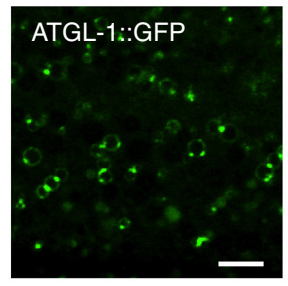

C

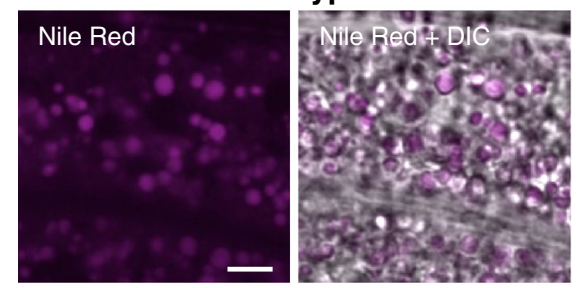

D

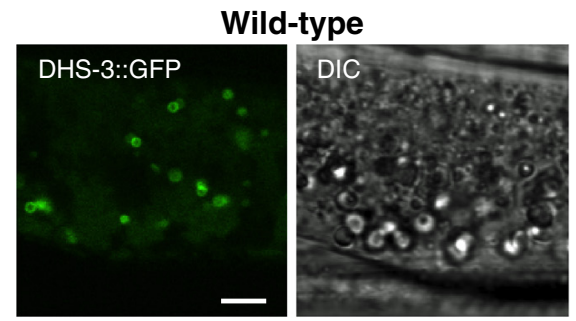

haf-4(gk240)
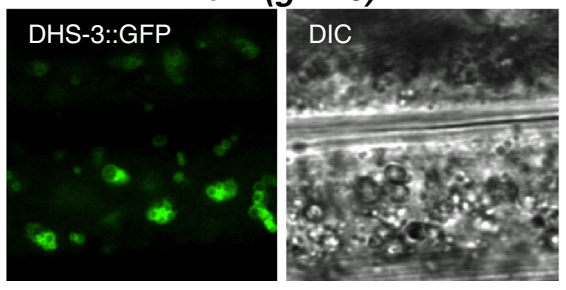
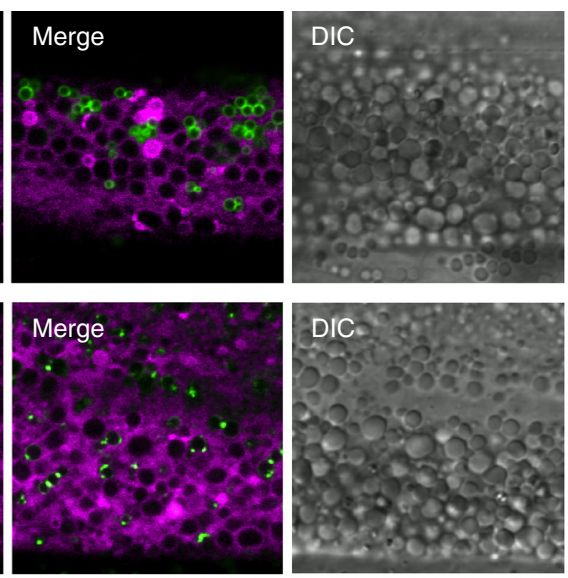

haf-4(ok1042) haf-9(gk23)

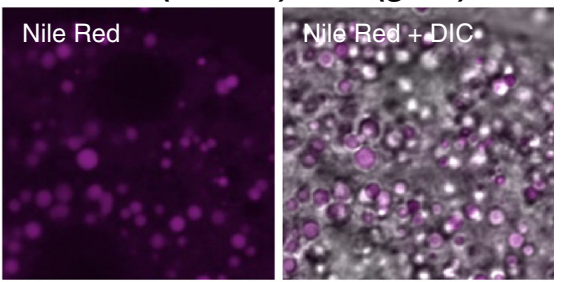

haf-4(ok1042)

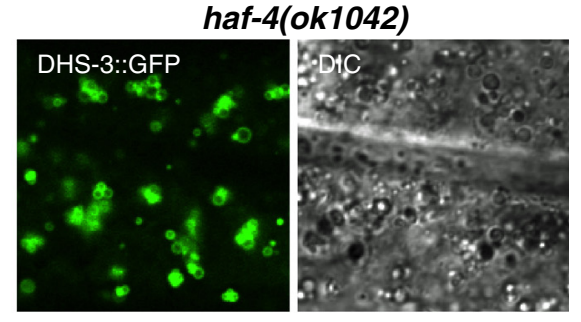

haf-9(gk23)
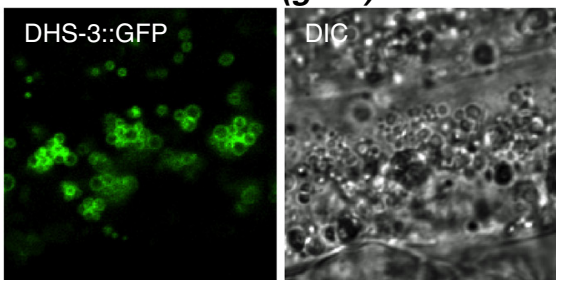

Fig. 1 HAF-4- and HAF-9-localizing intestinal organelles are distinct from lipid droplets. $\mathbf{a}$ and $\mathbf{b}$ Comparison of the localization of HAF-9::mCherry with the lipid droplet markers DHS-3::GFP (a) and ATGL-1::GFP (b) in Is[dhs-3::GFP]/Is[haf-9::mCherry] and Is[atgl-1::GFP];/s[haf-9::mCherry], respectively. DHS-3::GFP or ATGL-1::GFP (green), HAF-9::mCherry (magenta), the merged images, and the corresponding DIC images are presented. DHS-3::GFP and HAF-9::mCherry did not localize to the surface of identical intestinal granules. c Nile Red staining of the wild-type N2 (left) and the haf4(ok1042) haf-9(gk23) double-mutant worms (right). Nile Red (magenta) and the merged images with DIC are presented. Nile Red-positive intestinal granules were intact even in the absence of the HAF-4- and HAF-9-localizing organelles. $\mathbf{d}$ Expression of the lipid droplet marker DHS-3::GFP in the wild-type (top left), haf-4(ok1042) (top right), haf-4(gk240) (bottom left), and haf-9(gk23) (bottom right) worms. DHS-3::GFP-positive lipid droplets did not decrease in these mutants. Bars, $5 \mu \mathrm{m}$

LMP-1::mRFP-positive granules (Additional file 1: Figure S5). Furthermore, the uptake of VIT-2::GFP in embryos was unaffected in the haf-4 haf-9 double mutant (Fig. 2c). These results suggest that the HAF4- and HAF-9-localizing organelles are irrelevant to yolk protein transport.
HAF-4- and HAF-9-localizing organelles are not acidified gut granules

Although the HAF-4- and HAF-9-localizing organelles are not acidified, LMP-1, a homolog of mammalian LAMPs, also localizes to these organelles, suggesting a potential relationship with the lysosomes [17]. HAF-4 
A
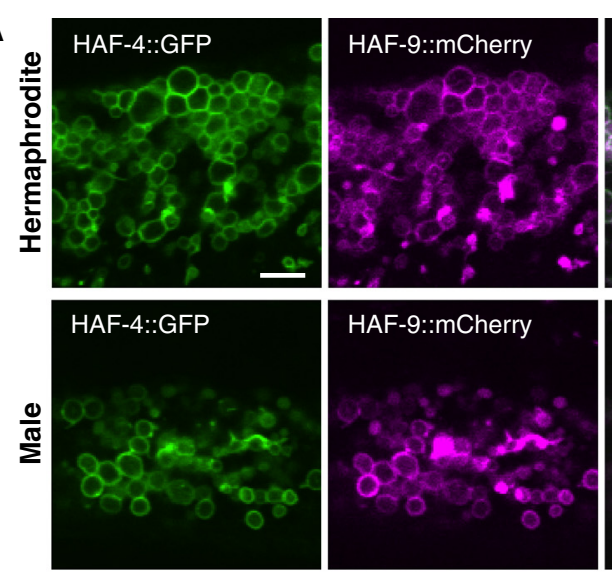

B
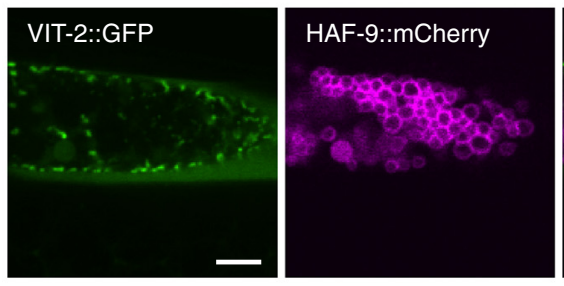

C

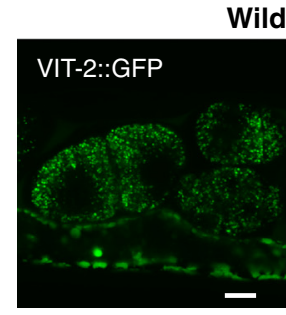

Wild-type

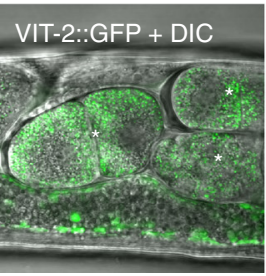

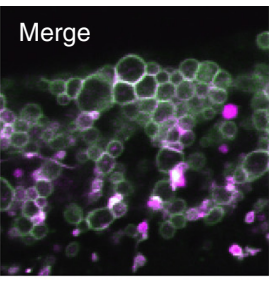
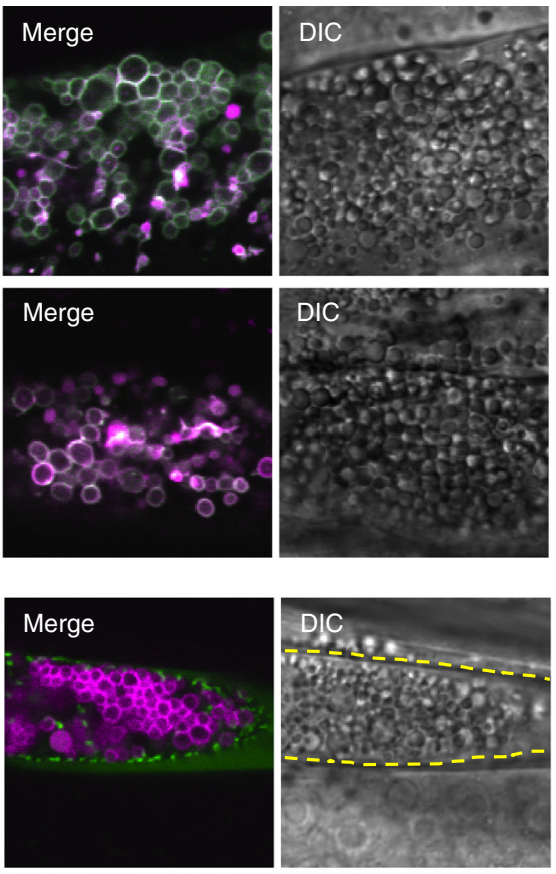

haf-4(ok1042) haf-9(gk23)
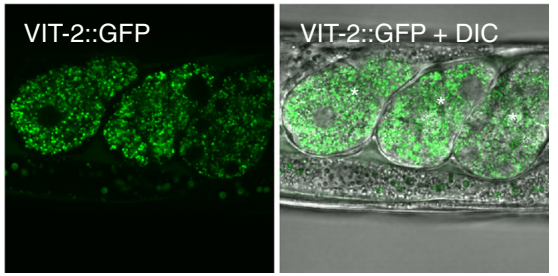

Fig. 2 HAF-4- and HAF-9-localizing intestinal organelles are irrelevant to the storage and distribution of yolk granules. a Localization of HAF-4:GFP (green) and HAF-9:mCherry (magenta) to the intestinal granules in the day-1 adult hermaphrodite (top) and male (bottom) worms of Is[haf-4::GFP]; Is[haf-9::mCherry]. The merged images and the corresponding DIC images are also presented. HAF-4::GFP and HAF-9::mCherry colocalized on the surface of intestinal granules not only in the hermaphrodites but also in the males. Bar, 5 mm. b Comparison of the localization of HAF-9::mCherry with VIT-2::GFP in Is[vit2::GFP];/IS[haf-9::mCherry]. VIT-2::GFP (green), HAF-9::mCherry (magenta), the merged image, and the corresponding DIC image are presented. VIT-2::GFP did not localize to the HAF-9::mCherry-positive intestinal granules. Dashed lines indicate the boundary of the intestine. Bar, 5 um. c The localization of VIT-2::GFP in the wild-type (left) and haf-4(ok1042)haf-9(gk23) (right) worms. VIT-2:GFP and the merged images with corresponding DIC images are presented. Developing embryos positive for VIT-2::GFP are indicated by asterisks. Distribution of VIT-2::GFP to the embryos was unaltered even in the mutant worms. Bar, $10 \mu \mathrm{m}$

and HAF-9 do not localize to organelles with autofluorescent materials that are characteristic of gut granules, and haf-4 and haf-9 were found to be unnecessary for the formation of gut granules. To investigate the relationship between these organelles and gut granules in more detail, we compared the localization of HAF-4 and HAF-9 with that of GLO-1, a small GTPase required for the biogenesis of gut granules. GLO-1::GFP localized to the surface of autofluorescent granules but not to the Nile Red-positive lipid droplets (Additional file 1: Figure S6). In adult worms expressing both GLO-1::GFP and HAF-9::mCherry, the mCherry-edged intestinal granules (arrows in Fig. 3a) and the mCherry-internalized intestinal granules (arrowheads in Fig. 3a) were observed, the latter of which are thought to be lysosomes or lysosomerelated organelles accumulating mCherry in their lumen. Although GLO-1::GFP localized to the surface of the mCherry-internalized granules, it did not localize to the mCherry-edged intestinal granules where HAF9::mCherry was localized on the membrane (Fig. 3a and Additional file 1: Figure S13c). Similarly, GLO-1::GFP did not localize to the LMP-1::mRFP-edged intestinal granules (Additional file 1: Figure S7). We also investigated the localization of HAF- 4 and HAF-9 in the glo- 1 mutant. The localization of HAF-4::GFP and HAF9::mCherry to the surface of intestinal granules was observed even in the glo-1(zu391) mutants (Fig. 3b and Additional file 1: Figure S13d-e), as was the localization 

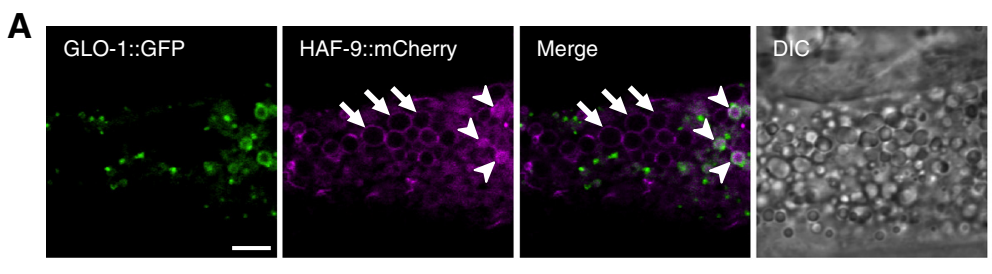

\section{B}
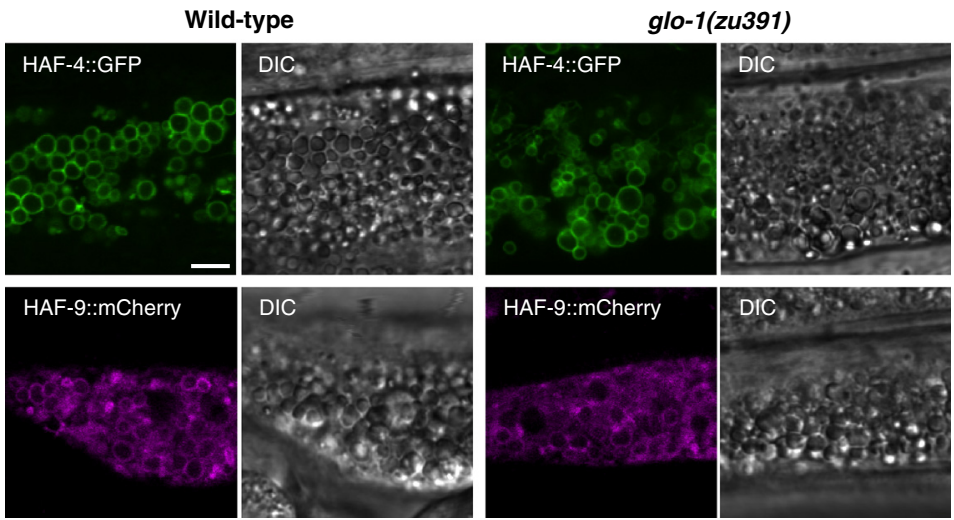

Fig. 3 HAF-4- and HAF-9-localizing intestinal organelles are not acidified gut granules. a Comparison of the localization of HAF-9::mCherry with GLO-1::GFP in Is[ges-1p::g/o-1::GFP];/s[haf-9::mCherry]. GLO-1::GFP (green), HAF-9::mCherry (magenta), the merged image, and the corresponding DIC image are presented. GLO-1:GFP did not localize to the mCherry-edged intestinal granules (arrows). Arrowheads indicate the accumulation of mCherry in the gut granules. b The localization of HAF-4::GFP (top) and HAF-9::mCherry (bottom) in the wild-type (left) and glo-1 (zu391) (right) worms. The corresponding DIC images are also presented. HAF-4::GFP-positive and HAF-9::mCherry-positive intestinal granules were observed even in the mutant worms. Bars, $5 \mu \mathrm{m}$

of DHS-3::GFP (Additional file 1: Figure S8). These results indicate that the HAF-4- and HAF-9-localizing organelles are not acidified gut granules.

\section{HAF-4- and HAF-9-localizing organelles are associated with the endocytic pathway}

Although the HAF-4- and HAF-9-localizing organelles are prominent intestinal granules in late larval and young adult worms, they are distinct from acidified gut granules and lipid droplets. Furthermore, HAF-4::GFP and HAF9::mCherry were not localized to either the peroxisome marker-positive organelles or the mitochondrion markerpositive organelles (Additional file 1: Figure S9). Therefore, what are these organelles?

The localization of HAF-4 and HAF-9 to the enlarged vacuoles in $p p k-3(n 2668)$ mutants suggests their involvement in the endocytic pathway [17]. PPK-3 regulates terminal lysosome maturation and the $p p k-3$ mutation results in the enlargement of vacuoles where LMP-1 and RAB-7 localize [21]. We compared the localization of HAF-9::mCherry with that of the late-endosomal and lysosomal marker GFP::RAB-7, which did not localize to autofluorescent granules or Nile Red-positive lipid droplets (Additional file 1: Figure S10). Although the GFP::RAB-7 signal showed a more dispersed pattern than that of HAF-9::mCherry, GFP::RAB-7 and HAF9::mCherry colocalized to the surface of intestinal granules (Fig. 4a and Additional file 1: Figure S13f). Both the altered localization of GFP::RAB-7 in the haf-4 and haf-9 mutants and the localization of GFP::RAB-7 to the enlarged organelles in the $1 m p-1(n r 2045)$ mutant strongly support the localization of GFP::RAB-7 to the HAF-4and HAF-9-localizing organelles. The localization of HAF-4::GFP and HAF-9::mCherry to the surface of intestinal granules was severely impaired in the rab-7 mutant (Fig. 4c and Additional file 1: Figure S13g-h), as was that of LMP-1::mRFP (Additional file 1: Figure S11). In contrast, the autofluorescent granules and DHS-3::GFP-positive lipid droplets were not affected in the rab-7 mutant (Additional file 1: Figure S12).

\section{Discussion}

We investigated the relationships among the intestinal granular organelles of $C$. elegans using two approaches. The first was through comparison of the localization of fluorescent protein-tagged proteins and fluorescently visualizable materials inside the organelles, facilitating the identification of organelles to clarify the interrelationships in their formation processes. The other approach involved investigating how a defect in a subset of intestinal organelles affected other subsets, which could help to determine whether or not these organelles require each other for their biogenesis and function. 


\section{A}
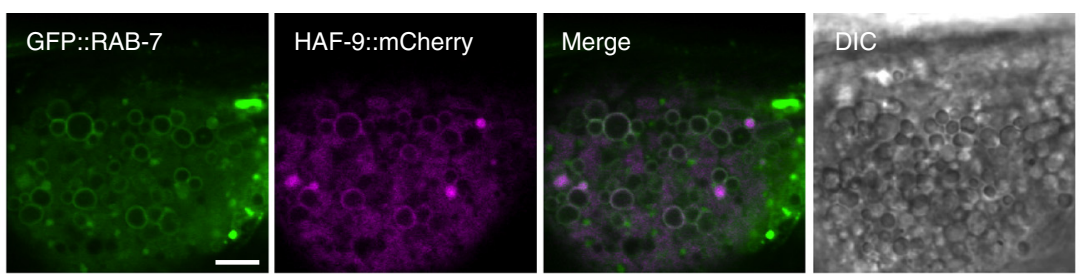

B

GFP::RAB-7

DIC
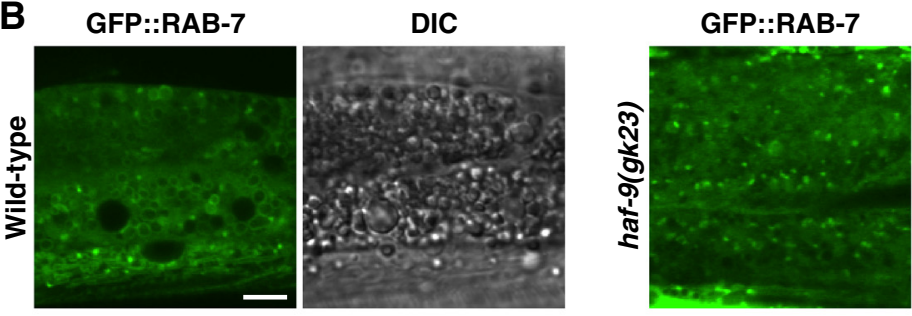

DIC
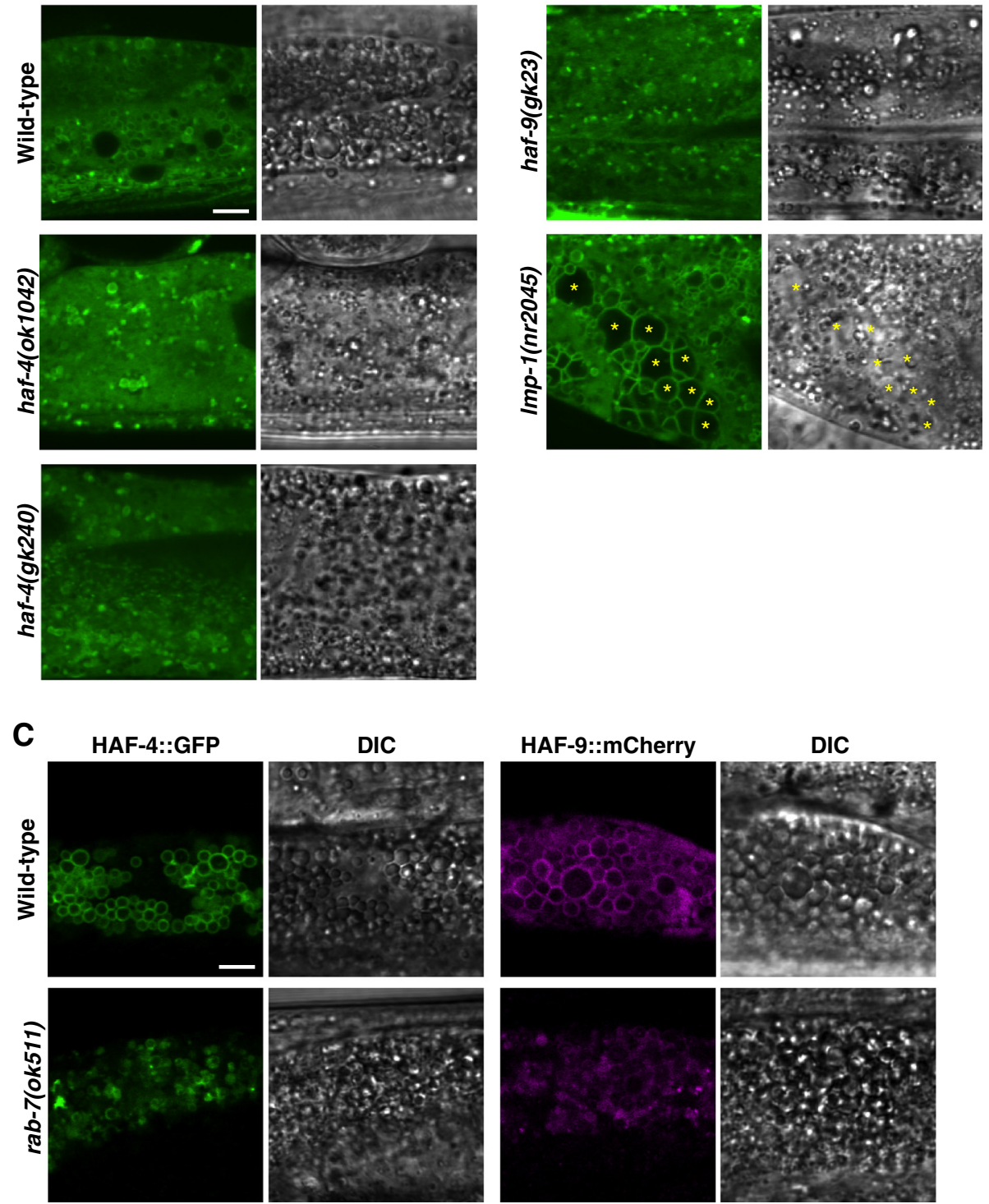

Fig. 4 HAF-4- and HAF-9-localizing organelles are associated with the endocytic pathway. a Co-localization of GFP::RAB-7 and HAF-9::mCherry in Is [haf-9::mCherry];Ex[GFP::rab-7]. GFP::RAB-7 (green) HAF-9::mCherry (magenta), the merged image, and the corresponding DIC image are presented. b Localization of GFP::RAB-7 in the wild-type (top left) haf-4(ok1042) (middle left), haf-4(gk240) (bottom left), haf-9(gk23) (top right), and Imp-1 (nr2045) (middle right). GFP::RAB-7 (green) and the corresponding DIC images are presented. In the haf-4 and haf-9 mutants, exhibiting defects in the HAF-4- and HAF-9-localizing organelles, the number of intestinal granules with GFP fluorescence on their surface decreased. In Imp-1(nr2045), GFP fluorescence was detected on the surface of the enlarged vacuole (asterisks). c Subcellular localization of HAF-4::GFP (left) and HAF-9::mCherry (right) in the wild-type (top) and rab-7(ok511) (bottom). The corresponding DIC images are also presented. HAF-

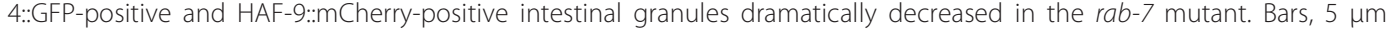


These investigations clearly showed that the HAF-4- and HAF-9-localizing organelles are not lipid droplets, based on the lack of Nile Red stainability and DHS-3::GFP localization, and that they are not required for the biogenesis and lipid storage of lipid droplets. It was also confirmed that the organelles are not yolk granules and are not necessary for the transport of yolk protein to developing oocytes. The involvement of these organelles in the storage or distribution of nutrients has been suggested, because haf-4 and haf-9 mutants, which are defective in organelle biogenesis, produced phenotypes with slow growth and reduced brood size. However, the organelles seem to be irrelevant for the storage and distribution of lipids and yolk protein. The storage of polypeptides and/or carbohydrates is a possible candidate for their function. In particular, the storage of peptides seems to be a plausible function, because HAF-4 and HAF-9 are believed to transport peptides from the cytosol to the lumen of the organelles, based on their homology with the mammalian peptide transporter TAPL [17].

Furthermore, HAF-4- and HAF-9-positive intestinal granules did not overlap with GLO-1-positive granules, and the HAF-4- and HAF-9-localizing organelles and acidified gut granules did not require each other for their biogenesis, suggesting that they are distinct organelles that are formed by different pathways. Experiments using GFP::RAB-7 and the rab-7 mutants demonstrated that RAB-7 associates with HAF-4- and HAF-9-localizing organelles and participates in their biogenesis. The small GTPase RAB-7 is a key regulator of the endocytic pathway, and replacement of RAB- 5 with RAB- 7 occurs when early endosomes mature into late endosomes [22]. Therefore, our results strongly suggest that the HAF-4- and HAF-9-localizing organelles are formed through the maturation or fusion of RAB-7-positive late endosomes. Transport substrates of HAF-4 and HAF-9 may possibly be necessary for the maturation or fusion process. The multivesicular structure observed in the $\operatorname{lmp}-1$ mutant under a transmission electron microscope also suggested a defect in the endocytic pathway [17]. More interestingly, the biogenesis of acidified gut granules requires the Rab38 homologue GLO-1 but not RAB-7 [9], providing further evidence that HAF-4- and HAF-9-localizing organelles and acidified gut granules are irrelevant. Mammalian Rab38 associates with melanosomes and platelet dense granules and is necessary for their biogenesis [23, 24]. In contrast to the acidified gut granules, which are lysosomerelated organelles, HAF-4- and HAF-9-localizing organelles are thought to be more related to the canonical lysosomes in terms of their biogenesis, even though they are not acidified and are much larger than the average lysosome (approximately $2 \mu \mathrm{m}$ versus $0.05-0.5 \mu \mathrm{m}$ in diameter). The possibility that they are residual bodies is also unlikely because autofluorescent materials known as age pigments, which are characteristic of residual bodies [25], are attributed to acidified gut granules but not to HAF-4and HAF-9-localizing organelles.

\section{Conclusions}

Although the physiological function of the HAF-4- and HAF-9-localizing organelles remains elusive despite their abundance in intestinal cells, our data demonstrate that they are distinct intestinal organelles associated with the endocytic pathway. The storage of polypeptides and/or carbohydrates is a possible candidate for their function. Uncovering the specific function of the organelles and their relationship with other intracellular compartments would provide insight into the organelle diversity to support the multifunctionality of C. elegans intestinal cells.

\section{Methods}

\section{General methods and establishment of mutant and transgenic strains}

The maintenance, husbandry, and genetic crosses of $C$. elegans were performed according to the standard protocols described by Brenner [26]. Strains were cultured at $20{ }^{\circ} \mathrm{C}$ unless otherwise noted. Bristol strain N2 was used as the standard wild-type strain, and the following mutant and transgenic strains were obtained from the Caenorhabditis Genetics Center (University of Minnesota): haf-4(ok1042), haf-4(gk240), haf-9(gk23), lmp-1(nr2045), glo-1(zu391), rab-7(ok511), hjIs67[atgl-1::GFP + mec-7::m RFP], bIs1[vit-2::GFP + rol-6(su1006)], hjIs9[ges-1p::glo1::GFP + unc-119(+)], hjIs37[vha-6p::mRFP-PTS1 + Cbr-u $n c-119(+)$ ], hjIs8[ges-1p::GFP-PTS1], and zcIs17[ges-1::GF $P(m i t)]$. The transgenic strains Is [haf-4::GFP + rol-6(su10 06)], Is[haf-9::GFP + rol-6(su1006)], Is[haf-9::mCherry + rol-6(su1006)], and Is[ges-1p::lmp-1::mRFP + rol-6(su100 6)] were established as described by Kawai et al. [17] and Tanji et al. [18]. Is[dhs-3::GFP] was provided by Dr. Liu (Institute of Biophysics, Chinese Academy of Sciences, Beijing, China).

To generate the GFP::rab-7 construct in which enhanced green fluorescent protein (EGFP) is fused inframe at the N-terminus of RAB-7, three DNA fragments were polymerase chain reaction (PCR)-amplified and fused as follows. A 1.6-kb DNA fragment (corresponding to the promoter region of $\mathrm{rab}-7$ ) and a 1.9-kb DNA fragment (corresponding to the coding sequence and 3' untranslated region of $\mathrm{rab}-7$ ) were PCR-amplified with the primer sets rab-7_A\#F3/rab-7_A\#R3 and rab-7_A\#F1/ rab-7_A\#R1, respectively. A 0.7-kb sequence of the EGFP coding region was PCR-amplified with the primer set rab7_A\#F2/ rab-7_A\#R2. These DNA fragments were fused by PCR sewing with the primer set rab-7_A\#F3/ rab7_A\#R1, and subsequently cloned between the NotI and BamHI sites of the pBlueScript vector.

The primer sequences used for establishment of the constructs were as follows: 


\begin{tabular}{ll}
\hline rab-7_A\#F1 & gcatggacgagctgtacaagATGTCGGGAACCAGAAAGAAG \\
rab-7_A\#R1 & gcagcccgggggatccTTCCAGACGCCAATTGAGAG \\
rab-7_A\#F2 & AAAAGGCTTCCAGTGAACAAAAatggtgagcaagggcgagga \\
rab-7_A\#R2 & CTTCTाTCTGGTTCCCGACATcttgtacagctcgtccatgc \\
rab-7_A\#F3 & accgcggtggcggccgcATTCGCGCCATTACCTCAAA \\
rab-7_A\#R3 & tcctcgcccttgctcaccatTTTGTCACTGGAAGCCTाT \\
\hline
\end{tabular}

Microinjection of DNA into the C. elegans germ line was performed as described by Mello et al. [27] using pRF4 [rol-6(su1006)] as a selection marker. Integrant formation was performed according to Mitani's method [28]. Our study does not require any animal ethics approval.

\section{Nile Red staining}

The worms were washed with S-basal $(50 \mathrm{mM}$ potassium phosphate buffer $[\mathrm{pH} 6.0]$ containing $0.1 \mathrm{M} \mathrm{NaCl}$ ) and fixed with $0.5 \%$ paraformaldehyde in phosphate-buffered saline for $1 \mathrm{~h}$ at room temperature. Fixed worms were rinsed with $\mathrm{M} 9$ buffer (42 $\mathrm{mM} \mathrm{Na} \mathrm{NPO}_{4}, 22 \mathrm{mM}$ $\mathrm{KH}_{2} \mathrm{PO}_{4}, 86 \mathrm{mM} \mathrm{NaCl}, 1 \mathrm{mM} \mathrm{MgSO}, 0.02 \%$ gelatin) and stored at $4{ }^{\circ} \mathrm{C}$ until required. They were stained with $1 \mu \mathrm{g} /$ $\mathrm{mL}$ Nile Red in M9 buffer prepared from a stock solution ( $1 \mathrm{mg} / \mathrm{mL}$ Nile Red in acetone) for $15-30 \mathrm{~min}$ at room temperature.

\section{Optical microscopic observation}

The differential interference contrast (DIC) and autofluorescence images were obtained using an epifluorescence microscope (BX51; Olympus Corp., Tokyo, Japan) with DIC optics. Fluorescence images of GFP, mCherry, mRFP, and Nile Red were obtained using a confocal microscope (FV1000; Olympus Corp., Tokyo) with 473-nm or 559-nm laser excitation. Spectral scanning and unmixing were performed using the spectral deconvolution program in the FV1000 software FV10-ASW. For the unmixing from autofluorescence, autofluorescence was enhanced by preexposure to ultraviolet rays. All images were obtained using hermaphrodites on adult day 1 after anesthetization with $50 \mathrm{mM} \mathrm{NaN}_{3}$ in M9 buffer, unless otherwise indicated. The glo-1::GFP and GFP::rab-7 transgenic worms subjected to the microscopic investigation were reared at $16{ }^{\circ} \mathrm{C}$ for clearer identification. All images presented are of the middle part of the intestine (int3 to int7) unless otherwise noted, oriented with the anterior to the left. The presented images are the representatives of at least three animals.

\section{Additional file}

Additional file 1: Figure S1. Staining of Is[haf-4::GFP] (a) and Is[haf9::GFP] (b) with the lysochrome dye Nile Red after fixation. Figure S2. DHS-3-positive intestinal granules are not autofluorescent granules. Figure S3. $1 \mathrm{mp}-1$ is not required for the biogenesis of DHS-3::GFP- positive lipid droplets. Figure S4. VIT-2::GFP did not localize to either the autofluorescent granules or the lipid droplets. Figure S5. LMP1::mRFP did not localize to VIT-2::GFP-positive granules. Figure S6. GLO-1::GFP-positive granules are autofluorescent but not Nile Redpositive. Figure S7. LMP-1::mRFP did not localize to GLO-1::GFP-positive granules. Figure S8. glo-1 is not required for the biogenesis of DHS-3:.:GFPpositive lipid droplets. Figure S9. HAF-4 and HAF-9 did not localize to either the peroxisomes or the mitochondria. Figure S10. GFP::RAB-7 did not localize to either the autofluorescent granules or the Nile Red-positive lipid droplets. Figure S11. rab-7 is required for the biogenesis of LMP$1:: \mathrm{mCherry}$-positive granules. Figure S12. rab-7 is not required for the biogenesis of autofluorescent granules and DHS-3::GFP-positive lipid droplets. Figure S13. Other representative images. (PDF 5985 kb)

\section{Abbreviations}

ABC: ATP-binding cassette; DIC: Differential interference contrast; GFP: Green fluorescent protein; LAMP: Lysosome-associated membrane protein; PCR: Polymerase chain reaction; TAPL: Transporter associated with antigen processing-like.

\section{Competing interests}

The authors declare that they have no competing interests.

\section{Authors' contributions}

AOK conceived of and directed this study. TT, KN, HS, KGA, SM, and AOK contributed to the discussion of the experimental design and data analysis and wrote the paper. $\Pi$ T, KN, YKs, and MT performed the analysis of lipid droplets. KN and SH performed the analysis of VIT-2. TT, KN, YK, and SH performed the analysis of GLO-1. $\Pi$, KN, and KGA performed the analysis of RAB-7. All authors read and approved the final manuscript.

\section{Acknowledgements}

We thank Ms. Reiko Aoyama, Ms. Ayumi Hosoda, and Ms. Kikuko Kawano for their help throughout all stages of this work. We also thank Mr. Yoshio Hamada for his experimental support. We are grateful to Dr. Pingsheng Liu for providing Is[dhs-3::GFP]. Many strains were provided by the CGC, which is funded by the NIH Office of Research Infrastructure Programs (P40 OD010440).

This work was supported by JSPS KAKENHI [grant numbers 22590070 (AOK), 22790081 (HS), 23790154 (TT), 26860087 (TT), and 25460071 (AOK)] and by The Kao Foundation for Arts and Sciences (TT).

\section{Author details}

'Department of Immunobiology, School of Pharmacy, Iwate Medical University, 2-1-1 Nishi-tokuta, Yahaba, Shiwa-gun, Iwate 028-3694, Japan. 2Department of Physiology, School of Medicine, Tokyo Women's Medical University, 8-1 Kawada-cho, Shinjuku-ku, Tokyo 162-8666, Japan. ${ }^{3}$ Present address: Saitama University Brain Science Institute, 255 Shimo-okubo, Sakura-ku, Saitama 338-8570, Japan.

Received: 5 August 2015 Accepted: 3 December 2015

Published online: 27 January 2016

\section{References}

1. McGhee JD. The C. elegans intestine (March 27, 2007), WormBook ed. In: WormBook. The C. elegans Research Community. 2007. doi: 10.1895/wormbook.1.133.1, http://www.wormbook.org. Accessed 27 Oct 2015.

2. White J. The Anatomy. In: Wood WB, the Community of C. elegans Researchers, editors. The Nematode Caenorhabditis elegans. Cold Spring Harbor, New York: Cold Spring Harbor Laboratory Press; 1988. p. 81-122.

3. Clokey GV, Jacobson LA. The autofluorescent "lipofuscin granules" in the intestinal cells of Caenorhabditis elegans are secondary lysosomes. Mech Ageing Dev. 1986;35:79-94.

4. Hermann GJ, Schroeder LK, Hieb CA, Kershner AM, Rabbitts BM, Fonarev P, et al. Genetic analysis of lysosomal trafficking in Caenorhabditis elegans. Mol Biol Cell. 2005;16:3273-88.

5. Schroeder LK, Kremer S, Kramer MJ, Currie E, Kwan E, Watts JL, et al. Function of the Caenorhabditis elegans ABC transporter PGP-2 in the biogenesis of a lysosome-related fat storage organelle. Mol Biol Cell. 2007;18:995-1008. 
6. Currie E, King B, Lawrenson AL, Schroeder LK, Kershner AM, Hermann GJ. Role of the Caenorhabditis elegans multidrug resistance gene, $m r p-4$, in gut granule differentiation. Genetics. 2007;177:1569-82.

7. Rabbitts BM, Ciotti MK, Miller NE, Kramer M, Lawrenson AL, Levitte $\mathrm{S}$, et al. glo-3, a novel Caenorhabditis elegans gene, is required for lysosome-related organelle biogenesis. Genetics. 2008;180:857-71.

8. Hermann GJ, Scavarda E, Weis AM, Saxton DS, Thomas LL, Salesky R, et al. C. elegans BLOC-1 functions in trafficking to lysosome-related gut granules. PLOS ONE. 2012;7:e43043.

9. Delahaye JL, Foster OK, Vine A, Saxton DS, Curtin TP, Somhegyi H, et al. Caenorhabditis elegans HOPS and CCZ-1 mediate trafficking to lysosomerelated organelles independently of RAB-7 and SAND-1. Mol Biol Cell. 2014; 25:1073-96.

10. Roh HC, Collier S, Guthrie J, Robertson JD, Kornfeld K. Lysosome-related organelles in intestinal cells are a zinc storage site in C. elegans. Cell Metab. 2012;15:88-99.

11. Lee HJ, Zhang W, Zhang D, Yang Y, Liu B, Barker EL, et al. Assessing cholesterol storage in live cells and C. elegans by stimulated Raman scattering imaging of phenyl-Diyne cholesterol. Sci Rep. 2015;5:7930.

12. Folick A, Oakley HD, Yu Y, Armstrong EH, Kumari M, Sanor L, et al. Lysosomal signaling molecules regulate longevity in Caenorhabditis elegans. Science. 2015;347:83-6.

13. O'Rourke EJ, Soukas AA, Carr CE, Ruvkun G. C. elegans major fats are stored in vesicles distinct from lysosome-related organelles. Cell Metab. 2009;10: 430-5.

14. Zhang SO, Trimble R, Guo F, Mak HY. Lipid droplets as ubiquitous fat storage organelles in C. elegans. BMC Cell Biol. 2010;11:96.

15. Zhang $\mathrm{P}, \mathrm{Na} \mathrm{H}$, Liu Z, Zhang $\mathrm{S}$, Xue $\mathrm{P}$, Chen $\mathrm{Y}$, et al. Proteomic study and marker protein identification of Caenorhabditis elegans lipid droplets. Mol Cell Proteomics. 2012;11:317-28.

16. Mullaney BC, Ashrafi K. C. elegans fat storage and metabolic regulation. Biochim Biophys Acta. 2009;1791:474-8.

17. Kawai H, Tanji T, Shiraishi H, Yamada M, lijima R, Inoue T, et al. Normal formation of a subset of intestinal granules in Caenorhabditis elegans requires ATP-binding cassette transporters HAF-4 and HAF-9, which are highly homologous to human lysosomal peptide transporter TAP-like. Mol Biol Cell. 2009:20:2979-90.

18. Tanji T, Nishikori K, Shiraishi H, Maeda M, Ohashi-Kobayashi A. Co-operative function and mutual stabilization of the half ATP-binding cassette transporters HAF-4 and HAF-9 in Caenorhabditis elegans. Biochem J. 2013; 452:467-75.

19. Kimble J, Sharrock WJ. Tissue-specific synthesis of yolk proteins in Caenorhabditis elegans. Dev Biol. 1983;96:189-96.

20. Grant B, Hirsh D. Receptor-mediated endocytosis in the Caenorhabditis elegans oocyte. Mol Biol Cell. 1999;10:4311-26.

21. Nicot AS, Fares H, Payrastre B, Chisholm AD, Labouesse M, Laporte J. The phosphoinositide kinase PIKfyve/Fab1p regulates terminal lysosome maturation in Caenorhabditis elegans. Mol Biol Cell. 2006;17:3062-74.

22. Rink J, Ghigo E, Kalaidzidis Y, Zerial M. Rab conversion as a mechanism of progression from early to late endosomes. Cell. 2005;122:735-49.

23. Loftus SK, Larson DM, Baxter LL, Antonellis A, Chen Y, Wu X, et al. Mutation of melanosome protein RAB38 in chocolate mice. Proc Natl Acad Sci U S A 2002;99:4471-6.

24. Ambrosio AL, Boyle JA, Di Pietro SM. Mechanism of platelet dense granule biogenesis: study of cargo transport and function of Rab32 and Rab38 in a model system. Blood. 2012;120:4072-81.

25. Harman D. Lipofuscin and ceroid formation: the cellular recycling system. Adv Exp Med Biol. 1989;266:3-15.

26. Brenner S. The genetics of Caenorhabditis elegans. Genetics. 1974;77:71-94.

27. Mello CC, Kramer JM, Stinchcomb D, Ambros V. Efficient gene transfer in C. elegans: extrachromosomal maintenance and integration of transforming sequences. EMBO J. 1991;10:3959-70.

28. Mitani S. Genetic regulation of mec-3 gene expression implicated in the specification of the mechanosensory neuron cell types in Caenorhabditis elegans. Dev Growth Diff. 1995;37:551-7.

\section{Submit your next manuscript to BioMed Central and we will help you at every step:}

- We accept pre-submission inquiries

- Our selector tool helps you to find the most relevant journal

- We provide round the clock customer support

- Convenient online submission

- Thorough peer review

- Inclusion in PubMed and all major indexing services

- Maximum visibility for your research

Submit your manuscript at www.biomedcentral.com/submit 